IFUP-TH 70/96

\title{
Critical limit and anisotropy in the two-point correlation function of three-dimensional $\mathrm{O}(N)$ models.
}

\author{
Massimo Campostrini, Andrea Pelissetto, Paolo Rossi, and Ettore Vicari \\ Dipartimento di Fisica dell'Università and I.N.F.N., I-56126 Pisa, Italy
}

(May 22, 2018)

\begin{abstract}
In three-dimensional $\mathrm{O}(N)$ models, we investigate the low-momentum behavior of the two-point Green's function $G(x)$ in the critical region of the symmetric phase. We consider physical systems whose criticality is characterized by a a rotationally-invariant fixed point.

In non rotationally-invariant physical systems with $\mathrm{O}(N)$-invariant interactions, the vanishing of anisotropy in approaching the rotationally-invariant fixed point is described by a critical exponent $\rho$, which is universal and is related to the leading irrelevant operator breaking rotational invariance. At $N=\infty$ one finds $\rho=2.1 / N$ expansion and strong-coupling calculations show that, for all values of $N \geq 0, \rho \simeq 2$.

Non-Gaussian corrections to the universal low-momentum behavior of $G(x)$ are evaluated, and found to be very small.
\end{abstract}

PACS numbers: 05.70.Jk, 64.60.Fr, 75.10.Hk, 75.40.Cx 
Three-dimensional $\mathrm{O}(N)$ models describe many important critical phenomena in nature. We just mention that the case $N=3$ describes the critical properties of ferromagnetic materials. The case $N=2$ is related to the helium superfluid transition. The case $N=$ 1 (i.e., Ising-like systems) describes liquid-vapour transitions in classical fluids or critical binary fluids. Finally, the limit $N \rightarrow 0$ is related to dilute polymers.

The critical behavior of the two-point correlation function $G(x)$ of the order parameter is related to the phenomenon of critical scattering observed in many experiments, e.g. neutron scattering in ferromagnetic materials, light and X-rays in liquid-gas systems. In Born's approximation, the cross section $\Gamma_{f i}$ for incoming particles (i.e. neutrons or photons) of momentum $p_{i}$ and final outgoing momentum $p_{f}$ is proportional to the component $k=p_{f}-p_{i}$ of the Fourier transform of $G(x)$

$$
\Gamma_{f i} \propto \widetilde{G}(k), \quad k=p_{f}-p_{i} .
$$

As a consequence of the critical behavior of the two-point function $G(x)$ at $T_{c}$, which is

$$
\widetilde{G}(k) \sim \frac{1}{k^{2-\eta}},
$$

the cross section for $k \rightarrow 0$ (forward scattering) is observed to diverge as $T \rightarrow T_{c}$. When strictly at criticality, the relation (2) holds at all momentum scales $k \ll \Lambda$, where $\Lambda$ is a generic cut-off related to the microscopic structure of the statystical system. In the vicinity of the critical point, where the relevant correlation length $\xi$ is large but finite, the behavior (2) occurs for $\Lambda \gg k \gg 1 / \xi$.

We will specifically consider systems with an $\mathrm{O}(N)$-invariant Hamiltonian in the symmetric phase, i.e. where the $\mathrm{O}(N)$ symmetry is unbroken. Furthermore, we will only consider systems with a rotationally-symmetric fixed point. Interesting members of this class are systems defined on highly symmetric lattices, i.e. Bravais or two-point base lattices with a tetrahedral or larger discrete rotational symmetry.

Let us consider the second moment correlation length

$$
\xi_{G}^{2}=\frac{1}{6} \frac{\sum_{x}|x|^{2} G(x)}{\sum_{x} G(x)},
$$

and the mass scale $M_{G} \equiv 1 / \xi_{G}$. In the critical region of the symmetric phase and at low momentum, experiments show that $G(x)$ is well approximated by a Gaussian behavior, i.e.

$$
\frac{\widetilde{G}(0)}{\widetilde{G}(k)} \simeq 1+\frac{k^{2}}{M_{G}^{2}} .
$$

Our aim is to estimate the deviations from Eq. (4) in the critical region of the symmetric phase, i.e. for $0<T / T_{c}-1 \ll 1$, and in the low-momentum regime $k^{2} \lesssim M_{G}^{2}$. We focus on two quite different sources of deviations:

(i) Scaling corrections to Eq. (四), depending on the ratio $k^{2} / M_{G}^{2}$, and reflecting the non-Gaussian nature of the fixed point.

(ii) Non rotationally-invariant scaling violations, reflecting a microscopic anisotropy in the space distribution of the spins (assuming that no anisotropy is generated by their interaction). This phenomenon may be relevant, for example, in the study of ferromagnetic 
materials, where the atoms lie on the sites of a lattice, and anisotropy may be observed in neutron scattering experiments. In these systems anisotropy vanishes in the critical limit, and $G(x)$ approaches a rotationally-invariant form.

We mention that the effects of a breakdown of $\mathrm{O}(N)$ symmetry in the interactions have been widely considered in the literature [1].

Several approaches have been considered in order to study the critical behavior of the two-point function

$$
G(x)=\langle\vec{s}(x) \cdot \vec{s}(0)\rangle .
$$

In lattice $\mathrm{O}(N)$ non-linear $\sigma$ models, we have calculated the strong-coupling expansion of $G(x)$ up to 15 th order on the cubic lattice [2] and 21st order on the diamond lattice within the corresponding nearest-neighbor formulations,

$$
S_{L}=-N \beta \sum_{\text {links }} \vec{s}_{x_{l}} \cdot \vec{s}_{x_{r}}
$$

where $x_{l}, x_{r}$ indicate the sites at the ends of each link. Our conclusions are integrated and supported by results obtained from a $1 / N$ expansion of lattice $\mathrm{O}(N)$ models. Furthermore, we have analyzed the first nontrivial term of the $\epsilon$-expansion and of the $g$-expansion (i.e. expansion in the coupling at fixed dimensions $d=3$ ) of the two-point function within the corresponding $\phi^{4}$ formulation of $\mathrm{O}(N)$ models.

Our results substantially confirm experimental observations that scaling corrections to the Gaussian behavior (4) are very small. This point has been already discussed in the literature, essentially by $\epsilon$-expansion [3] and $1 / N$ expansion [4] in the continuum formulation of $\mathrm{O}(N)$ models. We present here rather accurate determinations of such corrections obtained by a strong-coupling analysis of $G(x)$.

We will show that the anisotropy of $G(x)$, for the class of systems we are considering, vanishes at the rotationally-invariant fixed point, with a behavior governed by a universal critical exponent $\rho$ : non-spherical moments (i.e. those which vanish when calculated on spherical functions) of $G(x)$ are depressed with respect to spherical moments carrying the same naive physical dimensions by a factor $\xi^{-\rho}$. From a field-theoretical point of view, anisotropy in space is due to non rotationally-invariant (but $\mathrm{O}(N)$ symmetric) irrelevant operators in the effective Hamiltonian, whose presence depends essentially on the symmetries of the physical system, or of the lattice formulation. Another possible origin is the coupling of the system to an external (anisotropic) source. The exponent $\rho$ is related to the critical effective dimension of the leading irrelevant operator breaking rotational invariance. On cubic-like lattices the leading operator has canonical dimension $d+2$. In the large- $N$ limit, where the canonical dimensions determine the scaling properties, one then finds $\rho=2$. $1 / N$ expansion and strong-coupling calculations show that, for all values of $N \geq 0, \rho$ remains close to its canonical value. One can also show that for the two-dimensional Ising model the exact result $\rho=2$ holds. Moreover, in the $1 / N$ expansion of two-dimensional models, one finds that, to $O(1 / N)$, the multiplicative logarithmic correction to the behavior $\sim \xi^{-2}$ is absent.

The technical details of our study will be reported in a separate extended paper. Here we only describe the general features of our analysis and the main numerical results. 
For definiteness let us consider the cubic lattice version of the models. We parametrize the two-point spin-spin function by a multipole expansion in the form

$$
\beta^{-1} \widetilde{G}^{-1}\left(k, M_{G}\right)=\sum_{l=0}^{\infty} g_{2 l}\left(y, M_{G}\right) Q_{2 l}(k),
$$

where $y \equiv k^{2} / M_{G}^{2}$, and $Q_{2 l}(k)$ are homogeneous functions of momenta of degree $2 l$ which are invariant under the symmetries of the lattice. Their expressions can be obtained from the fully symmetric traceless tensors of rank $2 l, T_{2 l}^{\alpha_{1} \ldots \alpha_{2 l}}(k)$, by considering all the cubicinvariant combinations [5]. Odd rank terms are absent in the expansion (7) because of the parity symmetry. Moreover, there is no rank-two term, due to the discrete rotational symmetry of the lattice. The first nontrivial function is

$$
Q_{4}(k)=\sum_{\mu} k_{\mu}^{4}-\frac{3}{5}\left(\sum_{\mu} k_{\mu}^{2}\right)^{2}
$$

$Q_{4}(k)$ corresponds to the leading irrelevant operator that breaks rotational invariance in the low-momentum expansion of the Hamiltonian. In the continuum notation this operator has the form

$$
O_{4}(x)=\vec{s}(x) \cdot Q_{4}(\partial) \vec{s}(x),
$$

which has canonical dimension five in $d=3$.

The scaling limit of Eq. (7) corresponds to taking $M_{G} \rightarrow 0$ while keeping the ratio $k / M_{G}$ finite. Hence we can parametrize the low-momentum behavior of $G^{-1}\left(k, M_{G}\right)$ in the critical region by

$$
\begin{aligned}
& \beta^{-1} \widetilde{G}^{-1}\left(k, M_{G}\right)=Z^{-1} \widehat{g}_{0}(y) M_{G}^{2}+\ldots \\
& \quad+Z_{4} Q_{4}\left(k / M_{G}\right) \widehat{g}_{4}(y) M_{G}^{4}+O\left(M_{G}^{6}\right)
\end{aligned}
$$

where we have dropped rotationally-invariant $O\left(M_{G}^{4}\right)$ terms which we are not interested in, and we have introduced the quantities

$$
Z^{-1}=\frac{g_{0}\left(0, M_{G}\right)}{M_{G}^{2}}, \quad Z_{4}=g_{4}\left(0, M_{G}\right)
$$

which, in the limit $M_{G} \rightarrow 0$, absorb all non-analytical dependence on $M_{G}$ of the corresponding terms. The functions $\widehat{g}_{0}(y)$ and $\widehat{g}_{4}(y)$ are universal, i.e., they do not depend on the specific form of the lattice hamiltonian. They possess a regular expansion around $y=0$ :

$$
\begin{aligned}
& \widehat{g}_{0}(y)=1+y+\sum_{i=2}^{\infty} c_{i} y^{i}, \\
& \widehat{g}_{4}(y)=1+\sum_{i=1}^{\infty} d_{i} y^{i} .
\end{aligned}
$$

In the limit $N \rightarrow \infty$, the models are strictly Gaussian and therefore all the coefficients $c_{i}$ and $d_{i}$ are zero, 
We consider the spherical moments

$$
m_{2 j}=\sum_{x}|x|^{2 j} G(x),
$$

and the leading non-spherical moments

$$
q_{4, j}=\sum_{x}|x|^{2 j} Q_{4}(x) G(x)
$$

which vanish if $G(x)$ is rotationally invariant. The critical exponent $\rho$, describing the vanishing of anisotropy, and the coefficients $c_{i}$ and $d_{i}$ of the low-momentum expansion of $\widehat{g}_{0}(y)$ and $\widehat{g}_{4}(y)$, can be determined by studying appropriate combinations of the above moments in the critical limit. In the critical region

$$
\frac{q_{4, m}}{m_{4+2 m}} \sim \frac{1}{\xi^{\rho}}
$$

where $q_{2, m}$ and $m_{4+2 m}$ have the same naive physical dimensions.

In the case of non-Gaussian fixed points, like those corresponding to the theory at finite $N, O_{4}(x)$ develops an anomalous dimension $\sigma$, which causes a departure from the Gaussian value of $\rho, \rho=2+\sigma . \sigma$ can be extracted by evaluating the ratio

$$
\frac{Z Z_{4}}{M_{G}^{2}}=\frac{g_{4}\left(0, M_{G}\right)}{g_{0}\left(0, M_{G}\right)} \sim M_{G}^{\sigma} .
$$

In turn this combination is easily estimated by taking the moment ratio $q_{4,0} / m_{2} \sim M_{G}^{\sigma}$. We have calculated the first non-trivial term of the $1 / N, \epsilon$ and $g$-expansions of $\sigma$ :

$$
\begin{gathered}
\sigma=\frac{32}{21 \pi^{2} N}+O\left(\frac{1}{N^{2}}\right), \\
\sigma=\frac{7}{20} \frac{(N+2)}{(N+8)^{2}} \epsilon^{2}+O\left(\epsilon^{3}\right), \\
\sigma=\widetilde{g}^{* 2} \frac{5408}{25515} \frac{(N+2)}{(N+8)^{2}}+O\left(\widetilde{g}^{* 3}\right)
\end{gathered}
$$

( $\widetilde{g}^{*}$ is the fixed-point value of the rescaled coupling: $\widetilde{g}=g(N+8) / 48 \pi[$ [ ] $)$. Strong-coupling estimates of $\sigma$ have been obtained by analyzing and comparing the available strong-coupling series of $q_{4,0}$ and $m_{2}$ on both cubic and diamond lattices. Universality between cubic and diamond lattice is substantially verified, although the analysis on the diamond lattice leads

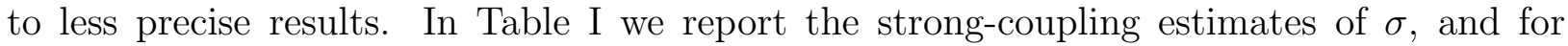
comparison its estimates from Eqs. (18), (19) and (20). For $\mathrm{O}(N)$ models, the values of $\sigma$ are very small in the whole range $N \geq 0$, thus indicating an essentially Gaussian behavior of this critical exponent. The comparison of the estimates of $\sigma$ from the various approaches we have considered is satisfactory.

The low-momentum critical behavior of the two-point Green's function, i.e. 


$$
\frac{\widetilde{G}(0)}{\widetilde{G}(k)} \rightarrow \widehat{g}_{0}(y),
$$

has been already investigated within an $\epsilon$-expansion of the $\phi^{4}$ formulations of $\mathrm{O}(N)$ models (up to $O\left(\epsilon^{2}\right)$ ) [3], and in the $1 / N$ expansion (up to $O(1 / N)$ ) [4]. The computations of the $O\left(1 / N^{2}\right)$ contribution to $\widehat{g}_{0}(y)$ is in progress. Moreover rough estimates of such corrections have been presented for $N=1$ and $N=3$ by analyzing strong-coupling expansion (up to 10th order) within various lattice formulations of the theory [3]. We have reconsidered the problem of determining the non-Gaussian corrections to $\widehat{g}_{0}(y)$ in the low-momentum regime, and those of $\widehat{g}_{4}(y)$, especially by a strong-coupling analysis. Furthermore we have calculated the first nontrivial contributions to $\widehat{g}_{4}(y)$ in the $1 / N, \epsilon$ and $g$-expansions, and of $\widehat{g}_{0}(y)$ in the $g$-expansion, and the corresponding coefficients of the expansion around $y=0$.

Our strong-coupling analysis leads to a satisfactory precision which, in the case of the coefficient $c_{i}$, considerably improves earlier calculations. This has been achieved essentially for two reasons: longer strong-coupling series are available, and, even more important, improved estimators have been employed. We indeed took special care in the choice of estimators for the physical quantities $c_{i}$ and $d_{i}$. This is very important from a practical point view: better estimators can greatly improve the stability of the extrapolation to the critical point. Our search for optimal estimators was guided by the knowledge of the large$N$ limit. We chose estimators which are perfect for $N=\infty$, i.e. do not present off-critical corrections to their critical value $c_{i}=d_{i}=0$ in the symmetric phase. In particular in the case of $c_{2}$ and $d_{1}$ and on the cubic lattice, we used the following estimators:

$$
\begin{aligned}
& e\left(c_{2}\right)=1-\frac{m_{4}}{120 m_{0}} M_{G}^{4}+\frac{1}{20} M_{G}^{2}, \\
& e\left(d_{1}\right)=2\left(1-\frac{q_{4,1}}{44 q_{4,0}} M_{G}^{2}+\frac{1}{44} M_{G}^{2}\right)
\end{aligned}
$$

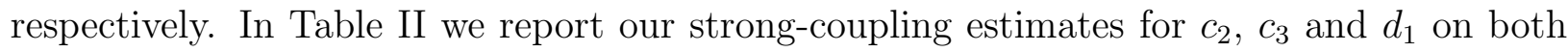
cubic and diamond lattice, obtained by evaluating at $\beta_{c}$ appropriate approximants (such as Padé and first order integral approximants) of the strong-coupling series of the corresponding estimators. One may notice that universality between cubic and diamond lattice is always confirmed. At large- $N$ the strong-coupling estimates of $c_{2}, c_{3}$ and $d_{1}$ compare very well with the corresponding $O(1 / N)$ formulas: $c_{2}=-0.004449 / N+\ldots, c_{3}=0.000134 / N+\ldots$, etc... 团, $d_{1}=-0.0020647 / N+\ldots, d_{2}=0.0000738 / N+\ldots$, etc... The comparison with the estimates from other approaches, which are reported in Table [1], is good.

All calculations agree in indicating that the inequality

$$
c_{i} \ll c_{2} \ll 1 \quad \text { for } \quad i \geq 3
$$

is satisfied for all $N$. These results show that, in the critical region of the symmetric phase, the two-point Green's function is substantially Gaussian in a large region around $k^{2}$, i.e. for $\left|k^{2} / M_{G}^{2}\right| \lesssim 1$, for all $N$ from zero to infinity. Another important consequence of the relation (24) is the possibility of evaluating the zero of $\widehat{g}_{0}(y)$ closest to the origin, $y_{0}$, to a rather good approximation by the relationship

$$
-y_{0} \simeq 1+c_{2} .
$$


The quantity $-y_{0}$ in turn is the scaling limit of the ratio of the correlation length defined in Eq. (3) with the "true" correlation length obtained from the damping factor in the exponential long-distance behavior of $G(x)$. Direct calculations of this ratio confirm the relationship (25). Similar results have been obtained in the two-dimensional $\mathrm{O}(N)$ models [6,7].

In general models defined on non-Bravais lattices such as the diamond lattice are not parity-invariant, and odd-rank operators are allowed in the corresponding expansion of the effective Hamiltonian. We finally discuss how space-parity violating terms, when they exist, vanish approaching the rotationally-invariant fixed point. This fact should be described by a critical exponent $\rho_{p}$ which should be universal in systems breaking parity at a microscopic level, such as ferromagnetic materials having the structure of a diamond lattice. $\rho_{p}$ can be evaluated on the nearest-neighbor formulation of $\mathrm{O}(N)$ models on the diamond lattice, which is not parity invariant. In the correspoding Gaussian theory, or the large- $N$ limit of $\mathrm{O}(N)$ models, one has $\rho_{p}=3$, indeed one finds that

$$
\frac{\sum x y z G(x, y, z)}{\sum G(x, y, z)} \longrightarrow \text { const. }
$$

in the critical limit. In general, for finite $N, \rho_{p}$ may differ from its Gaussian value. The strong-coupling analysis of the odd moments of $G(x)$ on the diamond lattice shows that the correction to the Gaussian value of $\rho_{p}$ is very small. We estimated that $0 \leq \rho_{p}-3 \lesssim 0.01$ for all $N \geq 0$. 


\section{REFERENCES}

[1] See for example J. Zinn-Justin, "Quantum Field Theory and Critical Phenomena", Clarendon Press, Oxford 1989, and reference therein.

[2] We mention that longer strong-coupling series, up to 19th order, of the lowest moments of $G(x)$ on the cubic and bcc lattices have been recently presented in P. Butera and M. Comi, Phys. Rev. B 52, 6185 (1995).

[3] M. E. Fisher and A. Aharony, Phys. Rev. Lett. 31, 1238 (1973); Phys. Rev. B 7, 2818 (1974).

[4] A. Aharony, Phys. Rev. B 7, 2834 (1974).

[5] For given rank $2 l$, there may be more than one invariant combination when $2 l \geq 12$ in three dimensions. Such a degeneracy is irrelevant to our discussion.

[6] M. Campostrini, A. Pelissetto, P. Rossi and E. Vicari, Phys. Rev. D 54, 1782 (1996).

[7] M. Campostrini, A. Pelissetto, P. Rossi and E. Vicari, Phys. Rev. B 54, 7301 (1996). 


\section{TABLES}

TABLE I. For various values of $N$, we report the estimates of $\sigma$ obtained by our strong-coupling analysis, and by the first nontrivial terms in the $1 / N$ expansion, $\epsilon$-expansion, and $g$-expansion. The errors diplayed in the strong-coupling estimates are a rough estimate of the uncertainty, which takes into account all the analyses we have performed.

\begin{tabular}{ccccc}
\hline \hline$N$ & s.c. & $1 / N$ exp & $\epsilon$-exp & $g$-exp \\
\hline \hline 0 & $0.00(1)$ & & 0.0109 & 0.0134 \\
1 & $0.01(1)$ & & 0.0130 & 0.0157 \\
2 & $0.02(1)$ & 0.0515 & 0.0140 & 0.0168 \\
3 & $0.03(2)$ & 0.0386 & 0.0145 & 0.0170 \\
4 & $0.03(2)$ & 0.0193 & 0.0147 & 0.0165 \\
8 & $0.02(1)$ & 0.0096 & 0.0137 & 0.0116 \\
16 & $0.009(3)$ & 0.0048 & 0.0109 & 0.0096 \\
32 & $0.004(2)$ & 0.0074 & 0.0054 \\
\hline \hline
\end{tabular}


TABLE II. Strong-coupling estimates of the coefficients $c_{2}$ and $c_{3}$ of the expansion of $\widehat{g}_{0}(y)$, and the coefficient $d_{1}$ of the expansion of $\widehat{g}_{4}(y)$. For comparison, we also report estimates from the first nontrivial terms in the $\epsilon$-expansion, $g$-expansion, and $1 / N$ expansion. The errors diplayed in the strong-coupling estimates should give an idea of the spread of the results from the various Padé-type and integral approximants we considered in our analysis.

\begin{tabular}{|c|c|c|c|c|}
\hline$N$ & & $c_{2}$ & $c_{3}$ & $d_{1}$ \\
\hline \multirow[t]{4}{*}{0} & cubic & $\left|c_{2}\right| \lesssim 2 \times 10^{-4}$ & $1.2(1) \times 10^{-5}$ & $1(1) \times 10^{-4}$ \\
\hline & diamond & $\left|c_{2}\right| \lesssim 1 \times 10^{-4}$ & $1.0(1) \times 10^{-5}$ & $-1.0(5) \times 10^{-4}$ \\
\hline & $\epsilon$-exp. & $-2.35 \times 10^{-4}$ & $0.60 \times 10^{-5}$ & $-1.11 \times 10^{-4}$ \\
\hline & $g$-exp. & $-3.12 \times 10^{-4}$ & $1.65 \times 10^{-5}$ & $-1.42 \times 10^{-4}$ \\
\hline \multirow[t]{4}{*}{1} & cubic & $-2.9(2) \times 10^{-4}$ & $1.1(1) \times 10^{-5}$ & $-1.7(5) \times 10^{-4}$ \\
\hline & diamond & $-3.1(2) \times 10^{-4}$ & $1.0(2) \times 10^{-5}$ & $-3(1) \times 10^{-4}$ \\
\hline & $\epsilon$-exp. & $-2.78 \times 10^{-4}$ & $0.71 \times 10^{-5}$ & $-1.31 \times 10^{-4}$ \\
\hline & $g$-exp. & $-3.66 \times 10^{-4}$ & $1.94 \times 10^{-5}$ & $-1.67 \times 10^{-4}$ \\
\hline \multirow[t]{4}{*}{2} & cubic & $-3.8(3) \times 10^{-4}$ & $1.1(1) \times 10^{-5}$ & $-2.3(2) \times 10^{-4}$ \\
\hline & diamond & $-4.2(3) \times 10^{-4}$ & $1.1(3) \times 10^{-5}$ & $-3(1) \times 10^{-4}$ \\
\hline & $\epsilon$-exp. & $-3.01 \times 10^{-4}$ & $0.77 \times 10^{-5}$ & $-1.42 \times 10^{-4}$ \\
\hline & $g$-exp. & $-3.90 \times 10^{-4}$ & $2.06 \times 10^{-5}$ & $-1.78 \times 10^{-4}$ \\
\hline \multirow[t]{4}{*}{3} & cubic & $-4.0(2) \times 10^{-4}$ & $1.1(2) \times 10^{-5}$ & $-2.5(2) \times 10^{-4}$ \\
\hline & diamond & $-4.6(4) \times 10^{-4}$ & $1.1(3) \times 10^{-5}$ & $-2.6(3) \times 10^{-4}$ \\
\hline & $\epsilon$-exp. & $-3.11 \times 10^{-4}$ & $0.79 \times 10^{-5}$ & $-1.46 \times 10^{-4}$ \\
\hline & $g$-exp. & $-3.95 \times 10^{-4}$ & $2.09 \times 10^{-5}$ & $-1.80 \times 10^{-4}$ \\
\hline \multirow[t]{5}{*}{4} & cubic & $-4.1(2) \times 10^{-4}$ & $1.2(1) \times 10^{-5}$ & $-2.5(2) \times 10^{-4}$ \\
\hline & diamond & $-4.7(2) \times 10^{-4}$ & $1.0(2) \times 10^{-5}$ & $-2.5(5) \times 10^{-4}$ \\
\hline & $\epsilon$-exp. & $-3.13 \times 10^{-4}$ & $0.80 \times 10^{-5}$ & $-1.48 \times 10^{-4}$ \\
\hline & $g$-exp. & $-3.85 \times 10^{-4}$ & $2.04 \times 10^{-5}$ & $-1.76 \times 10^{-4}$ \\
\hline & $1 / N$ exp. & $-11.12 \times 10^{-4}$ & $3.36 \times 10^{-5}$ & $-5.16 \times 10^{-4}$ \\
\hline \multirow[t]{5}{*}{8} & cubic & $-3.5(2) \times 10^{-4}$ & $1.0(2) \times 10^{-5}$ & $-2.1(2) \times 10^{-4}$ \\
\hline & diamond & $-4.0(1) \times 10^{-4}$ & $0.7(5) \times 10^{-5}$ & $-3(2) \times 10^{-4}$ \\
\hline & $\epsilon$-exp. & $-2.94 \times 10^{-4}$ & $0.75 \times 10^{-5}$ & $-1.38 \times 10^{-4}$ \\
\hline & $g$-exp. & $-2.70 \times 10^{-4}$ & $1.43 \times 10^{-5}$ & $-1.24 \times 10^{-4}$ \\
\hline & $1 / N$ exp. & $-5.56 \times 10^{-4}$ & $1.18 \times 10^{-5}$ & $-2.58 \times 10^{-4}$ \\
\hline \multirow[t]{5}{*}{16} & cubic & $-2.4(2) \times 10^{-4}$ & $0.70(5) \times 10^{-5}$ & $-1.4(2) \times 10^{-4}$ \\
\hline & diamond & $-2.65(5) \times 10^{-4}$ & $0.5(3) \times 10^{-5}$ & $-1.2(8) \times 10^{-4}$ \\
\hline & $\epsilon$-exp. & $-2.35 \times 10^{-4}$ & $0.60 \times 10^{-5}$ & $-1.11 \times 10^{-4}$ \\
\hline & $g$-exp. & $-2.25 \times 10^{-4}$ & $1.18 \times 10^{-5}$ & $-1.03 \times 10^{-4}$ \\
\hline & $1 / N$ exp. & $-2.78 \times 10^{-4}$ & $0.84 \times 10^{-5}$ & $-1.29 \times 10^{-4}$ \\
\hline \multirow[t]{5}{*}{$\overline{32}$} & cubic & $-1.44(5) \times 10^{-4}$ & $0.42(4) \times 10^{-5}$ & $-0.7(2) \times 10^{-4}$ \\
\hline & diamond & $-1.50(5) \times 10^{-4}$ & $0.3(2) \times 10^{-5}$ & $-0.5(3) \times 10^{-4}$ \\
\hline & $\epsilon$-exp. & $-1.60 \times 10^{-4}$ & $0.41 \times 10^{-5}$ & $-0.75 \times 10^{-4}$ \\
\hline & $g$-exp. & $-1.25 \times 10^{-4}$ & $0.66 \times 10^{-5}$ & $-0.57 \times 10^{-4}$ \\
\hline & $1 / N$ exp. & $-1.39 \times 10^{-4}$ & $0.42 \times 10^{-5}$ & $-0.64 \times 10^{-4}$ \\
\hline$\infty$ & & 0 & 0 & 0 \\
\hline
\end{tabular}

